\title{
Biomarkers for corneal graft rejection?
}

Eye (2009) 23, 247; doi:10.1038/eye.2008.308

Despite the many developments in immunosuppressive therapy, corneal graft rejection still is the leading cause for graft failure. ${ }^{1}$ This clinical scenario is a constant headache for ophthalmologists that regularly perform corneal transplants. Often long-term systemic immunosuppression is required with the undesirable consequences from systemic steroids or damage from the renal, hepatic, myelosuppressive effects from the use of other agents. Often a vexed clinical question after a rejection episode is when should such immunosuppressive regimes be stopped?

In this issue, Huang $e t a l^{2}$ describe an interesting observation that a gene involved in $\mathrm{T}$ helper 1 cell differentiation and cytokine production, Rac2, is increased in the peripheral blood in patients with corneal graft rejection. Most interesting is that the authors have tentatively shown that change in expression alters during a rejection episode, returning to 'normal' once the rejection process is in remission. The authors also show that there are
P Hossain

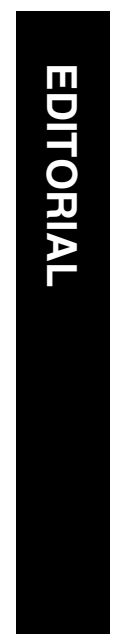

other genes involved, with the expression of CD18 and RhoA altered at the time of rejection.

Although Huang et $a l^{2}$ will need to perform further longitudinal studies to confirm their findings, these early results do lend to the possibility of a set of systemic biomarkers for transplant rejection. More crucially, they may have found markers that show when the rejection process is in remission!

So perhaps in the future, we will have an answer to our vexed question - when do we stop immunosuppressive therapy?

\section{References}

1 NHS blood and transplant Statistics and Audit Directorate. Transplant activity in the UK 2007-08. pp 44-62, http://www.uktransplant.org.uk/ukt/ statistics/transplant_activity_report/current_activity_ reports/ukt/tx_activity_report_2008_uk_pp44-62.pdf.

2 Huang M-C, Tullo AB, Hillarby MC. Increased Rac2 mRNA expression in peripheral blood during human corneal graft rejection. Eye 2009; 23: 461-469.
Eye Unit, Southampton General Hospital, University of Southampton, Southampton, Hampshire, UK

Correspondence: P Hossain, Eye Unit, Southampton General Hospital, University of Southampton, Tremona Road, Southampton, Hampshire SO16 6YD, UK Tel: 2380794570

Fax: 2380794120

E-mail: parwez@ soton.ac.uk 